\title{
The Menu-Induced Core of an Economy with an Excludable Public Good
}

\author{
Toshiyuki Hirai \\ Faculty of Economics, University of Toyama, Toyama, Japan \\ Email: thirai@eco.u-toyama.ac.jp
}

Received 1 February 2014; revised 5 March 2014; accepted 25 March 2014

Copyright (C) 2014 by author and Scientific Research Publishing Inc.

This work is licensed under the Creative Commons Attribution International License (CC BY). http://creativecommons.org/licenses/by/4.0/

(c) (i) Open Access

\begin{abstract}
This paper introduces a core concept in an economy with an excludable public good. In the economy, we assume that each coalition is allowed to achieve an allocation via a menu, a kind of a nonlinear price. Our core concept is called the menu-induced core that is defined as the set of allocations achievable by menus that are robust against all coalitional improvements achieved via menus. We show that the menu-induced core is nonempty. We also investigate certain properties of the menu-induced core that show the difference between the menu-induced core and the core defined in a standard way.
\end{abstract}

\section{Keywords}

\section{Menu-Induced Core, Excludable Public Good}

\section{Introduction}

This paper examines economies with excludable public goods. The public goods that we consider are those that admit partial exclusion: the amount that each agent consumes may vary from agent to agent. Tollways, pay-per-view TV programs, and public transportation are traditional examples. More recent examples include online commodities such as music and movie downloading services and access rights to databases through the internet. The early researches on such commodities are, for example, Oakland [1] and Drèze [2].

We consider stable allocations in cooperative decision situation of such an economy. In particular, we consider the core of the coalitional form of the economy. If we allow each coalition to achieve allocations with the individualized lump-sum payments, then the core essentially turns out to be that of Foley [3]. On the other hand, many examples of the excludable public goods are allocated through prices that may be nonlinear. For example, toll fare for the highway and a bundling sale of pay-per-view TV programs. According to this practice, we allow each coalition to achieve allocations via menus, which are a kind of nonlinear price. 
A nonlinear price is a kind of system. In the literature, several authors considered the core of economies where each coalition is allowed to achieve an allocation via a given system. Guesnerie and Oddou [4] [5] considered the core of a pure public good economy where each coalition achieves an allocation via proportional income tax. Spulber [6] considered the core of a production economy with increasing returns to scale where each coalition achieves an allocation via the average cost price. Hara [7] [8] considered the exchange economies where allocations are achieved via menus. Indeed, our concept of the coalitional improvement and the core is an application of his menu-induced improvement and the anonymous core to the economy with an excludable public good.

To justify such a way to achieve an allocation, we implicitly assume the same informational constraint as Hara [7] [8]. He assumed that each agent knows the distribution of the characteristics of the agents, but cannot identify who has which characteristic. Under such an informational constraint, it is difficult for the agent to find appropriate members of a coalition and an appropriate allocation achieved in the coalition. In this situation, employing a menu for achieving an allocation is legitimate for revealing the characteristics of the agents.

Our core concept is defined as the menu-induced core. It is defined as the set of allocations satisfying the following two conditions: the allocation is achievable within the grand coalition via a menu; and no coalition can achieve an allocation via a menu that makes each agent in the coalition better off. We show the nonemptiness of the menu-induced core and observe some properties, which clarify the difference between the menu-induced core and the standard Foley's core.

In the next section, we introduce the model of the economy with an excludable public good. In Section 3, we define the menu-induced core and prove its nonemptiness. Then, we investigate certain properties of the menu-induced core in Section 4. In the final section, we conclude with some remarks.

\section{The Economy with an Excludable Public Good}

We consider an economy consisting of $n$ agents, one private good, and one public good that is partially excludable. The set of agents is denoted by $N=\{1, \cdots, n\}$. A nonempty subset $S$ of $N$ is called a coalition. The set of all coalitions is denoted by $\mathcal{N}$.

A typical consumption of $i \in N$ is denoted by $\left(x_{i}, y_{i}\right) \in \mathbb{R}_{+}^{2}$, where $x_{i}$ and $y_{i}$ denote the amounts of the private good and the public good that $i$ consumes, respectively. The preferences of each $i \in N$ are represented by a utility function $u_{i}: \mathbb{R}_{+}^{2} \rightarrow \mathbb{R}$. For each $i \in N$, it is assumed that $u_{i}$ is continuous and monotone (i.e., $\left(x_{i}, y_{i}\right) \geq\left(x_{i}^{\prime}, y_{i}^{\prime}\right)$ implies $\left.u_{i}\left(x_{i}, y_{i}\right) \geq u_{i}\left(x_{i}^{\prime}, y_{i}^{\prime}\right)\right)$. Each $i \in N$ is endowed with $\omega_{i}>0$ of the private good.

Each coalition can access to an identical production technology that transforms the private good into the public good. The production technology is represented by a cost function $c: \mathbb{R}_{+} \rightarrow \mathbb{R}_{+}$. It is assumed that $c$ is continuous, monotone (i.e., $y \geq y^{\prime}$ implies $\left.c(y) \geq c\left(y^{\prime}\right)\right), \quad c(0)=0$, and $\lim _{y \rightarrow \infty} c(y)=\infty$.

For each $S \in \mathcal{N}$, an $S$-allocation is a tuple of the consumptions of agents in $S$. For each $S \in \mathcal{N}$, an $S$ allocation $\left(x^{S}, y^{S}\right)$ is said to be $S$-feasible iff $\sum_{i \in S}\left(\omega_{i}-x_{i}^{S}\right) \geq c\left(\max _{i \in S} y_{i}^{S}\right)$. Let $F(S)$ be the set of $S$ feasible allocations for each $S \in \mathcal{N}$. An $N$-allocation and an $N$-feasible allocation are simply called an allocation and a feasible allocation, respectively. Given an allocation $(x, y)$, an $S$-allocation $\left(x^{s}, y^{S}\right)$ is said to be an improvement upon $(x, y)$ for $S$ iff $\left(x^{S}, y^{S}\right) \in F(S)$ and $u_{i}\left(x_{i}^{S}, y_{i}^{S}\right)>u_{i}\left(x_{i}, y_{i}\right)$ for all $i \in S$.

Definition 1. An allocation $(x, y)$ is in the standard core iff $(x, y) \in F(N)$ and there exists no $S \in \mathcal{N}$ and $\left(x^{S}, y^{S}\right) \in F(S)$ that is an improvement upon $(x, y)$.

Obviously, the standard core is essentially equivalent to that of Foley [3] for economies with pure public goods.

\section{The Menu-Induced Core}

This section introduces the main concepts of this paper, which are defined as an application of the similar concepts of Hara [7] [8] with slight modifications. Then, its nonemptiness is proved.

The menu is defined as a subset $M$ of $\mathbb{R} \times \mathbb{R}_{+}$. It is a set of net consumptions: $(\ell, m) \in M$ means that $-\ell$ of the private good must be paid for consuming $m$ of the public good. Thus, the resulting consumption of $i \in N$ choosing $(\ell, m)$ is $\left(\omega_{i}+\ell, m\right)$.

For each $S \in \mathcal{N}$, let $V(S)$ be a set of $S$-allocations such that $(x, y) \in V(S)$ iff $(x, y) \in F(S)$ and 
there exists some $M \subset \mathbb{R} \times \mathbb{R}_{+}$such that for all $i \in S$, 1) $\left(x_{i}-\omega_{i}, y_{i}\right) \in M$ and 2) $u_{i}\left(x_{i}^{\prime}, y_{i}^{\prime}\right)>u_{i}\left(x_{i}, y_{i}\right)$ implies $\left(x_{i}^{\prime}-\omega_{i}, y_{i}^{\prime}\right) \notin M$. A tuple $(V(S))_{S \in \mathcal{N}}$ is called a menu-induced coalitional form of an economy.

An allocation $(x, y)$ is said to be menu-induced iff $(x, y) \in V(N)$. This definition describes the situation as follows: once a menu is proposed, each agent chooses the most preferable net consumption from the proposed menu. The menu can be proposed by an agent who may find it by oneself or consulting with an outside intervener.

Given an allocation $(x, y)$, a coalition $S$ has a menu-induced improvement upon $(x, y)$ iff there exists some $\left(x^{S}, y^{S}\right) \in V(S)$ such that $u_{i}\left(x_{i}^{S}, y_{i}^{S}\right)>u_{i}\left(x_{i}, y_{i}\right)$ for all $i \in N$. The improvement by a menu describes the following situation. Given an allocation, an agent proposes a menu to agents in the economy. Again, the proposing agent may find it by oneself or consulting with an outside intervener. Then, each agent responds to the menu when one can make oneself better off by choosing the most preferable net consumption from the menu. The coalition is formed among the agents who respond to the proposed menu. One may notice that the definition of the menu-induced improvement does not take the behavior of the agents outside the coalition into account. The original definition of Hara [7] [8] described the behavior of the agents outside the coalition as follows: no agent outside the coalition finds it more preferable to choose a net consumption from the menu. Thus, we may also impose the following condition on the menu-induced improvement $\left(x^{S}, y^{S}\right)$ upon a given allocation $(x, y)$ for $S$ : for all $j \in N \backslash S, u_{j}\left(x_{j}, y_{j}\right) \geq u_{j}\left(\omega_{j}+\ell, m\right)$ for any $(\ell, m) \in M$.

This condition is, however, redundant in our economy from the nonrivalry of the excludable public good. More precisely, for any allocation, there is a menu-induced improvement upon the allocation for $S \in \mathcal{N}$ if and only if there is a $T$-allocation with $S \subset T$ that satisfies both the definition of the menu-induced improvement and the additional condition. Therefore, imposing the additional condition does not change the nature of the menu-induced core that is defined below.

Definition 2. An allocation $(x, y)$ is said to be in the menu-induced core iff $(x, y) \in V(N)$ and there exist no $S \in \mathcal{N}$ and $\left(x^{S}, y^{S}\right) \in V(S)$ such that $\left(x^{S}, y^{S}\right)$ is a menu-induced improvement upon $(x, y)$ for $S$.

Now, we prove the nonemptiness of the menu-induced core.

Theorem 1. The menu-induced core is nonempty.

Proof. For any $q \geq 0$, define

$$
U(q)=\left\{(x, y) \in V(N) \mid \forall i \in N, x_{i} \leq \omega_{i} \text { and } u_{i}\left(x_{i}, y_{i}\right) \geq u_{i}\left(\omega_{i}, q\right)\right\} .
$$

Further, define

$$
\begin{aligned}
& Y=\left\{(x, y) \in F(N) \mid x_{i} \leq \omega_{i}, \forall i \in N\right\} ; \\
& Z=\left(\bigcup_{q \geq 0} U(q)\right) \cap Y .
\end{aligned}
$$

Clearly, $Z \neq \varnothing$ since $\left(\omega_{1}, \cdots, \omega_{n}, 0, \cdots, 0\right) \in U(0) \cap Y$. It can be easily confirmed that both $Y$ and $Z$ are compact sets by the assumptions of $u_{i}$ and $c$.

Define $Q(x, y)=\{q \geq 0 \mid(x, y) \in U(q)\}$ for each $(x, y) \in Z$. Note that $Q(x, y)$ is a nonempty interval of $\mathbb{R}_{+}$for any $(x, y) \in Z$ by definition.

First, assume that there exists some $\left(x^{\prime}, y^{\prime}\right) \in Z$ such that $Q\left(x^{\prime}, y^{\prime}\right)$ is unbounded above. Then, by the monotonicity of $u_{i}$,

$$
u_{i}\left(x_{i}^{\prime}, y_{i}^{\prime}\right) \geq u_{i}\left(x_{i}, y_{i}\right)
$$

for all $i \in N$ and any $\left(x_{i}, y\right) \in \mathbb{R}_{+}^{2}$ with $x_{i} \leq \omega_{i}$. We claim that $\left(x^{\prime}, y^{\prime}\right)$ is in the menu-induced core.

Suppose that there exist some $S \in \mathcal{N}$ and a menu-induced improvement $\left(x^{S}, y^{S}\right)$ upon $\left(x^{\prime}, y^{\prime}\right)$ for $S$. Then, $u_{i}\left(x_{i}^{S}, y_{i}^{S}\right)>u_{i}\left(x_{i}^{\prime}, y_{i}^{\prime}\right)$ for all $i \in S$. By (1), it must be $x_{i}^{S}>\omega_{i}$ for all $i \in S$. It follows that $0 \leq c\left(y^{S}\right) \leq \sum_{i \in S}\left(\omega_{i}-x_{i}^{S}\right)<0$ from the $S$-feasibility of $\left(x^{S}, y^{S}\right)$, a contradiction. Hence $\left(x^{\prime}, y^{\prime}\right)$ is in the menu-induced core.

Next, assume that $Q(x, y)$ is bounded for any $(x, y) \in Z$. We claim that $Q(x, y)$ is closed for any $(x, y) \in Z$. Fix an arbitrary $(\tilde{x}, \tilde{y}) \in Z$. Let $\left(q^{k}\right)_{k=1}^{\infty}$ be a sequence taken from $Q(\tilde{x}, \tilde{y})$ that converges to $\tilde{q}$. Then, we have $u_{i}\left(\tilde{x}_{i}, \tilde{y}_{i}\right) \geq u_{i}\left(\omega_{i}, q^{k}\right)$ for all $i \in \bar{N}$ and for all $k$. It follows from the continuity of $u_{i}$ that 
$u_{i}\left(\tilde{x}_{i}, \tilde{y}_{i}\right) \geq u_{i}\left(\omega_{i}, \tilde{q}\right)$ for all $i \in N$. Thus, in conjunction with the boundedness, $Q(x, y)$ is a compact set for any $(x, y) \in Z$.

Thus, we can define $\bar{q}(x, y)=\max \{q \mid q \in Q(x, y)\}$ for any $(x, y) \in Z$. We claim that $\bar{q}: Z \rightarrow \mathbb{R}_{+}$is an upper semi-continuous function. It suffices to show that $P(q)=\{(x, y) \in Z \mid q \in Q(x, y)\}$ is closed for any $q \geq 0$ by the definition of $\bar{q}$. Fix an arbitrary $\hat{q} \geq 0$. Let $\left(\left(x^{k}, y^{k}\right)\right)_{k=1}^{\infty}$ be a sequence taken from $P(\hat{q})$ that converges to $(\hat{x}, \hat{y})$. Then, we have $u_{i}\left(x_{i}^{k}, y_{i}^{k}\right) \geq u_{i}\left(\omega_{i}, \hat{q}\right)$ for all $i \in N$ and for all $k$. It follows from the continuity of $u_{i}$ that $u_{i}(\hat{x}, \hat{y}) \geq u_{i}\left(\omega_{i}, \hat{q}\right)$ for all $i \in N$. Thus, $(\hat{x}, \hat{y}) \in P(\hat{q})$. Hence $\bar{q}$ is an upper semi-continuous function.

Since $Z$ is a compact set and $\bar{q}: Z \rightarrow \mathbb{R}_{+}$is an upper semi-continuous function, there exists some $\left(x^{*}, y^{*}\right) \in Z$ such that $\bar{q}\left(x^{*}, y^{*}\right) \geq \bar{q}(x, y)$ for any $(x, y) \in Z$. We claim that $\left(x^{*}, y^{*}\right)$ is in the menuinduced core.

Let $q^{*}=\bar{q}\left(x^{*}, y^{*}\right)$. Suppose that there exist some $T \in \mathcal{N}$ and a menu-induced improvement $(\tilde{x}, \tilde{y})$ upon $\left(x^{*}, y^{*}\right)$ for $T$. By definition, $u_{i}\left(\tilde{x}_{i}, \tilde{y}_{i}\right)>u_{i}\left(x_{i}^{*}, y_{i}^{*}\right) \geq u_{i}\left(\omega_{i}, q^{*}\right)$ for all $i \in T$. Define $T^{\prime}=\left\{i \in T \mid \tilde{x}_{i} \leq \omega_{i}\right\}$. Then, $c\left(\max _{i \in T^{\prime}} \tilde{y}_{i}\right) \leq c\left(\max _{i \in T} \tilde{y}_{i}\right) \leq \sum_{i \in T}\left(\omega_{i}-\tilde{x}_{i}\right) \leq \sum_{i \in T^{\prime}}\left(\omega_{i}-\tilde{x}_{i}\right)$. Thus, $\left(\left(\tilde{x}_{i}, \tilde{y}_{i}\right)_{i \in T^{\prime}}\right)$ is still a menuinduced improvement upon $\left(x^{*}, y^{*}\right)$ for $T^{\prime}$ by its construction.

Then, $\tilde{y}_{i}>q^{*}$ for all $i \in T^{\prime}$ by the monotonicity of $u_{i}$. Define

$$
M=\left\{\left(\tilde{x}_{i}-\omega_{i}, \tilde{y}_{i}\right) \mid i \in T^{\prime}\right\} \cup\left\{\left(0, q^{*}+\varepsilon\right)\right\},
$$

where $\varepsilon>0$ is a sufficiently small real number so that $u_{i}\left(\tilde{x}_{i}, \tilde{y}_{i}\right)>u_{i}\left(\omega_{i}, q^{*}+\varepsilon\right)$ for all $i \in T^{\prime}$. Define

$$
\left(\bar{x}_{i}, \bar{y}_{i}\right)= \begin{cases}\left(\tilde{x}_{i}, \tilde{y}_{i}\right) & \text { if } i \in T^{\prime} \\ \left(\omega_{i}, 0\right)+\left(\ell_{i}, m_{i}\right) & \text { if } i \notin T^{\prime}\end{cases}
$$

where $\left(\ell_{i}, m_{i}\right) \in \arg \max _{(\ell, m) \in M} u_{i}\left(\omega_{i}+\ell, m\right)$ for each $i \notin T^{\prime}$. Note that $\bar{x}_{i} \leq \omega_{i}$ for all $i \in N$ since $\ell \leq 0$ for all $(\ell, m) \in M$ by its construction.

We confirm that $(\bar{x}, \bar{y}) \in V(N)$. The feasibility follows from $c\left(\max _{i \in N} \bar{y}_{i}\right)=c\left(\max _{i \in T^{\prime}} \tilde{y}_{i}\right) \leq \sum_{i \in T^{\prime}}\left(\omega_{i}-\tilde{x}_{i}\right) \leq \sum_{i \in N}\left(\omega_{i}-\bar{x}_{i}\right)$. By the construction of $(\bar{x}, \bar{y}), \quad\left(\bar{x}_{i}-\omega_{i}, \bar{y}\right) \in M$ for all $i \in N$. By the definitions of $(\tilde{x}, \tilde{y})$ and $M, u_{i}\left(\bar{x}_{i}, \bar{y}_{i}\right) \geq u_{i}\left(\omega_{i}+\ell, m\right)$ for any $(\ell, m) \in M$ and all $i \in N$. Hence $(\bar{x}, \bar{y}) \in V(N)$. Moreover, $u_{i}\left(\bar{x}_{i}, \bar{y}_{i}\right) \geq u_{i}\left(\omega_{i}, q^{*}+\varepsilon\right)$ for all $i \in N$ since $\left(0, q^{*}+\varepsilon\right) \in M$. Thus, $(\bar{x}, \bar{y}) \in U\left(q^{*}+\varepsilon\right) \cap Y$. This contradicts the definition of $q^{*}$. Hence $\left(x^{*}, y^{*}\right)$ is in the menu-induced core.

Note that we require neither the quasi-concavity of the utility functions nor the convexity of the cost function.

Note also that the proof of Theorem 1 applies the idea of Mas-Colell [9], which provides an alternative proof of a result of Champsaur [10]: the core of the coalitional game derived from an economy with one private good and one pure public good is nonempty. One may, therefore, consider that Theorem 1 is not surprising. However, the menu-induced core is generally much different from the standard core, which will be discussed in the next section.

\section{Observations}

In this section, we observe some properties of the menu-induced core, and discuss the difference from the standard core. One straightforward property from the definition is the symmetry of the allocations. Two agents $i$ and $j$ are symmetric iff $u_{i}(z)=u_{j}(z)$ for any $z \in \mathbb{R}_{+}^{2}$ and $\omega_{i}=\omega_{j}$. An allocation $(x, y)$ is symmetric iff $u_{i}\left(x_{i}, y_{i}\right)=u_{i}\left(x_{j}, y_{j}\right)$ for any symmetric agents $i$ and $j$. It can be easily confirmed that any allocation in the standard core may not be symmetric.

Property 1. Any allocation in the menu-induced core is symmetric.

The next property shows that the coalitional form of our economy may not satisfy the following usual 
property. The coalitional form of an economy $(V(S))_{S \in \mathcal{N}}$ is said to be superadditive at $N$ iff for all disjoint $S, T \in \mathcal{N}$, for any $\left(x^{S}, y^{S}\right) \in V(S)$ and any $\left(x^{T}, y^{T}\right) \in V(T)$, there exists some $(x, y) \in V(S \cup T)$ such that $u_{i}\left(x_{i}, y_{i}\right) \geq u_{i}\left(x_{i}^{S}, y_{i}^{S}\right)$ for all $i \in S$ and $u_{i}\left(x_{i}, y_{i}\right) \geq u_{i}\left(x_{i}^{T}, y_{i}^{T}\right)$ for all $i \in T$.

Property 2. $(V(S))_{S \in \mathcal{N}}$ may not be superadditive at $N$.

The following example shows Property 2.

Example 1. Let $N=\{1, \cdots, k, k+1\} \quad(k>1)$. Let $u_{i}\left(x_{i}, y_{i}\right)=\min \left\{x_{i}, \alpha y_{i}\right\}$ for all $i=1, \cdots, k$ and $u_{k+1}\left(x_{k+1}, y_{k+1}\right)=\min \left\{\alpha x_{k+1}, y_{k+1}\right\}$, where $\alpha>1$ is sufficiently large. Let $\omega_{i}=\bar{\omega}$ for all $i \in N$ and $c(y)=y$. Clearly, $\left(\frac{\alpha \bar{\omega}}{\alpha+1}, \frac{\bar{\omega}}{\alpha+1}\right) \in V(\{1\})$ and $\left(\frac{k \bar{\omega}}{\alpha+k}, \cdots, \frac{k \bar{\omega}}{\alpha+k} ; \frac{\alpha k \bar{\omega}}{\alpha+k}, \cdots, \frac{\alpha k \bar{\omega}}{\alpha+k}\right) \in V(\{2, \cdots, k+1\})$. We have $u_{1}\left(\frac{\alpha \bar{\omega}}{\alpha+1}, \frac{\bar{\omega}}{\alpha+1}\right)=\frac{\alpha \bar{\omega}}{\alpha+1}, u_{i}\left(\frac{k \bar{\omega}}{\alpha+k}, \frac{\alpha k \bar{\omega}}{\alpha+k}\right)=\frac{k \bar{\omega}}{\alpha+k}$ for all $i=2, \cdots, k$ and $u_{k+1}\left(\frac{k \bar{\omega}}{\alpha+k}, \frac{\alpha k \bar{\omega}}{\alpha+k}\right)=\frac{\alpha k \bar{\omega}}{\alpha+k}$.

Let $(x, y) \in V(N)$, induced by a menu $M$, such that $u_{1}\left(x_{1}, y_{1}\right) \geq \frac{\alpha \bar{\omega}}{\alpha+1}$ and $u_{k+1}\left(x_{k+1}, y_{k+1}\right) \geq \frac{\alpha k \bar{\omega}}{\alpha+k}$. Then, $\left\{(\ell, m),\left(\ell^{\prime}, m^{\prime}\right)\right\} \subset M$ for some $(\ell, m) \geq\left(-\frac{\bar{\omega}}{\alpha+1}, \frac{\bar{\omega}}{\alpha+1}\right)$ and some $\left(\ell^{\prime}, m^{\prime}\right) \geq\left(-\frac{\alpha \bar{\omega}}{\alpha+k}, \frac{\alpha k \bar{\omega}}{\alpha+k}\right)$. Then, it must be $x_{i} \geq \frac{\alpha \bar{\omega}}{\alpha+1}$ for each $i=1, \cdots, k, \quad x_{k+1} \geq \frac{k \bar{\omega}}{\alpha+k}$ and $y_{k+1} \geq \frac{\alpha k \bar{\omega}}{\alpha+k}$. Thus,

$$
\sum_{i \in N}\left(\bar{\omega}-x_{i}\right)-c\left(\max _{i \in N} y_{i}\right) \leq k\left(\frac{\bar{\omega}}{\alpha+1}\right)+\frac{k \bar{\omega}}{\alpha+k}-\frac{\alpha k \bar{\omega}}{\alpha+k}=\frac{k \bar{\omega}\left(\alpha+k+1-\alpha^{2}\right)}{(\alpha+1)(\alpha+k)} .
$$

Thus, the most LHS of (2) is negative since $\alpha$ is sufficiently large. In this case, $(x, y)$ is not feasible. Hence the coalitional form of the economy may not be superadditive.

Property 2 shows that our coalitional form is quite different from those in the literature. The coalitional form of an economy with one pure public good and one private good is known to be ordinary convex (see for example Peleg [11] for the definition of the ordinary convexity), which is a stronger condition than the superadditivity. See also Ichiishi [12]. On the other hand, Guesnerie and Oddou [4] [5] considered a coalitional form of an economy with one pure public good and one private good where each coalition is allowed to achieve an allocation through a proportional income tax. They showed that this coalitional form may not be superadditive and the core may be empty, while the menu-induced core is always nonempty in spite of the nonsuperadditivity.

The next property shows that there is a case where the menu-induced core is a subset of the standard core.

Property 3. If all agents are symmetric, then the menu-induced core is included in the standard core.

Proof. Assume that all agents are symmetric. Fix an arbitrary $(x, y)$ that is in the menu-induced core. For each $i \in N$, define $f_{i}(z)=u_{i}\left(\omega_{i}-\frac{c(z)}{n}, z\right)$ for any $z \in \mathbb{R}_{+}$.

We claim that $u_{i}\left(x_{i}, y_{i}\right) \geq \max _{z \in \mathbb{R}_{+}} f_{i}(z)$ for all $i \in N$. Suppose that there exists some $j \in N$ such that $u_{j}\left(x_{j}, y_{j}\right)<\max _{z \in \mathbb{R}_{+}} f_{j}(z)$. Then, by the symmetry of the agents, $u_{i}\left(x_{i}, y_{i}\right)<\max _{z \in \mathbb{R}_{+}} f_{i}(z)$ for all $i \in N$. Let $z^{*} \in \arg \max _{z \in \mathbb{R}_{+}} f_{i}(z)$ for all $i \in N$. Note that such $z^{*}$ exists by the symmetry of the agents. Then, we can easily confirm that $(\bar{x}, \bar{y})$ where $\bar{x}_{i}=\omega_{i}-\frac{z^{*}}{n}$ and $\bar{y}_{i}=z^{*}$ for all $i \in N$ is a menu-induced improvement upon $(x, y)$. This contradicts that $(x, y)$ is in the menu-induced core. Hence $u_{i}\left(x_{i}, y_{i}\right) \geq$ $\max _{z \in \mathbb{R}_{+}} f_{i}(z)$ for all $i \in N$.

Then, we show that $(x, y)$ is in the standard core. Suppose that there exist some $S \in \mathcal{N}$ and an $S$ - 
feasible allocation $\left(x^{S}, y^{S}\right)$ that is an improvement upon $(x, y)$ for $S$. Let $y^{\prime}=\max _{i \in S} y_{i}^{S}$. Since $u_{i}\left(x_{i}, y_{i}\right) \geq$ $\max _{z \in \mathbb{R}_{+}} f_{i}(z) \quad$ for $\quad$ all $i \in N, \quad u_{i}\left(x_{i}^{S}, y_{i}^{S}\right)>u_{i}\left(x_{i}, y\right) \geq u_{i}\left(\omega_{i}-\frac{c\left(y^{\prime}\right)}{n}, y^{\prime}\right) \quad$ for all $i \in N$. Thus, $x_{i}^{S}>\omega_{i}-\frac{c\left(y^{\prime}\right)}{n}$ for all $i \in S$. Then, $\sum_{i \in S}\left(\omega_{i}-x_{i}^{S}\right)<\frac{|S|}{n} c\left(y^{\prime}\right) \leq c\left(y^{\prime}\right)$, contradicting that $\left(x^{S}, y^{S}\right) \in F(S)$.

Hecne $(x, y)$ is in the standard core.

On the other hand, there is a case where the menu-induced core is disjoint with the standard core.

Property 4. The intersection of the menu-induced core and the standard core may be empty.

The following example shows Property 4, which is a modification of an example in Guesnerie and Oddou [5].

Example 2. Let $N=\{1, \cdots, 2 k\}, u_{i}\left(x_{i}, y_{i}\right)=x_{i}^{1-\alpha} y_{i}^{\alpha}$ for $i=1, \cdots, k$ and $u_{j}\left(x_{j}, y_{j}\right)=x_{j}^{\alpha} y_{j}^{1-\alpha}$ for $j=k+1, \cdots, 2 k$, where $0<\alpha<1 / 2$, and $\omega_{i}=1$ for all $i \in N$. The cost function is represented by $c(y)=y$.

At any allocation $(x, y)$ in the standard core, it can be easily confirmed that $y_{i}=\max _{j \in N} y_{j}$ for all $i \in N$ since the utility functions are continuous and strictly increasing in the interior of $\mathbb{R}_{+}^{2}$.

Let $(x, y)$ be an allocation in the standard core. If $(x, y)$ is a menu-induced allocation, then it must be $x_{i}=x_{j}$ for all $i, j \in N$ since $y_{i}=y_{j}$ and $\omega_{i}=\omega_{j}=1$ for all $i, j \in N$. Thus, there exists some $\left.z \in\right] 0,1[$ such that $\left(x_{i}, y_{i}\right)=(1-z, 2 k z)$ for all $i \in N$.

Let us consider two menus $\{(-\alpha, \alpha k)\}$ and $\{(-(1-\alpha),(1-\alpha) k)\}$. If at least $k$ agents prefer $(1-\alpha, \alpha k)$ $((\alpha,(1-\alpha) k)$, respectively) to $(1-z, 2 k z)$, then $(x, y)$ is not in the menu-induced core. Thus, if $(x, y)$ is in the menu-induced core, it must be both

$$
\begin{gathered}
(1-z)^{\alpha}(2 k z)^{1-\alpha} \geq \alpha^{\alpha}((1-\alpha) k)^{1-\alpha} \Leftrightarrow\left(\frac{1-z}{\alpha}\right)^{\alpha}\left(\frac{2 z}{1-\alpha}\right)^{1-\alpha} \geq 1 ; \\
(1-z)^{1-\alpha}(2 k z)^{\alpha} \geq(1-\alpha)^{1-\alpha}(\alpha k)^{\alpha} \Leftrightarrow\left(\frac{1-z}{1-\alpha}\right)^{1-\alpha}\left(\frac{2 z}{\alpha}\right)^{\alpha} \geq 1 .
\end{gathered}
$$

However, there exists no $z \in] 0,1[$ that simultaneously satisfies (3) and (4) if $\alpha$ is sufficiently small. Thus, in this example, the intersection of the standard core and the menu-induced core is empty.

By Property 3 and 4, there is no general relationship between the menu-induced core and the standard core.

\section{Concluding Remarks}

This paper defined the menu-induced core and showed its nonemptiness in an economy with an excludable public good. We also discussed some properties of the menu-induced core. One remaining problem is the evaluation of the efficiency of the menu-induced core. In general, the menu-induced core fails to achieve the Pareto efficiency, which may be caused by the underlying informational constraints. We may consider the extent of the inefficiency, or efficiency evaluation under the similar informational constraints.

Another remaining problem is to design a mechanism that implements the allocations in the menu-induced core. In the economy with an excludable public good, some mechanisms are proposed. For example, Moulin [13] proposed the serial cost sharing mechanism, and Moldovanu [14] and Bag and Winter [15] proposed mechanisms that implement the standard core. However, all of them do not implement the menu-induced core. We leave these problems for future research.

\section{Acknowledgements}

I am grateful to an anonymous reviewer and Mikio Nakayama for their helpful comments and advices. I am also grateful for the financial supports by JSPS Grant-in-aid for Young Scientists (B) 22730155 and JSPS Grant-in-aid for Scientific Research (B) 24310110.

\section{References}

[1] Oakland, W. (1974) Public Goods, Perfect Competition, and Underproduction. Journal of Political Economy, 82, 927-939. http://dx.doi.org/10.1086/260247

[2] Drèze, J.H. (1980) Public Goods with Exclusion. Journal of Public Economics, 13, 5-24. 
http://dx.doi.org/10.1016/0047-2727(80)90020-1

[3] Foley, D. (1970) Lindahl's Solution and the Core of an Economy with Public Goods. Econometrica, 38, 66-72. http://dx.doi.org/10.2307/1909241

[4] Guesnerie, R. and Oddou, C. (1979) On Economic Games Which Are Not Necessarily Superadditive. Economics Letters, 3, 301-306. http://dx.doi.org/10.1016/0165-1765(79)90001-6

[5] Guesnerie, R. and Oddou, C. (1981) Second-Best Taxation as a Game. Journal of Economic Theory, 25, 67-91. http://dx.doi.org/10.1016/0022-0531(81)90017-X

[6] Spulber, D.F. (1986) The Second Best Core. International Economic Review, 30, 623-631. http://dx.doi.org/10.2307/2526779

[7] Hara, C. (2002) The Anonymous Core of an Exchange Economy. Journal of Mathematical Economics, 38, 91-116. http://dx.doi.org/10.1016/S0304-4068(02)00066-6

[8] Hara, C. (2005) Bargaining Set and Anonymous Core without the Monotonicity Assumption. Journal of Mathematical Economics, 41, 545-556. http://dx.doi.org/10.1016/j.jmateco.2004.12.001

[9] Mas-Colell, A. (1980) Remarks on the Game-Theoretic Analysis of a Simple Distribution of Surplus Problem. International Journal of Game Theory, 9, 125-140. http://dx.doi.org/10.1007/BF01781368

[10] Champsaur, R.J. (1975) How to Share the Cost of a Public Good? International Journal of Game Theory, 4, $113-129$. http://dx.doi.org/10.1007/BF01780629

[11] Peleg, B. (1986) A Proof That the Core of an Ordinal Convex Game Is a von Neumann-Morgenstern Solution. Mathematical Social Sciences, 11, 83-87. http://dx.doi.org/10.1016/0165-4896(86)90006-5

[12] Ichiishi, T. (1993) The Cooperative Nature of the Firm. Cambridge University Press, Cambridge.

[13] Moulin, H. (1994) Serial Cost Sharing of Excludable Public Goods. Review of Economic Studies, 61, 305-325. http://dx.doi.org/10.2307/2297983

[14] Moldovanu, B. (1996) The Production and Cost-Sharing of an Excludable Public Good. Economic Theory, 7, 531-539. http://dx.doi.org/10.1007/BF01213665

[15] Bag, P.K. and Winter, E. (1999) Simple Subscription Mechanisms for Excludable Public Goods. Journal of Economic Theory, 87, 72-94. http://dx.doi.org/10.1006/jeth.1998.2497 